\title{
Analysis and Detection of a Target Gas System Based on TDLAS \& LabVIEW
}

\author{
Fawzi Alorifi \\ Electrical Engineering Department, \\ College of Engineering, Al Imam \\ Mohammad Ibn Saud Islamic University \\ (IMSIU), Riyadh, Saudi Arabia \\ fsorifi@imamu.edu.sa
}

\author{
Sidi M. Ahmed Ghaly \\ Electrical Engineering Department, \\ College of Engineering, Al Imam \\ Mohammad Ibn Saud Islamic University \\ (IMSIU), Riyadh, Saudi Arabia \\ smghaly@imamu.edu.sa
}

\author{
Mohamed Y. Shalaby \\ Electrical Engineering Department, \\ College of Engineering, Al Imam \\ Mohammad Ibn Saud Islamic University \\ (IMSIU), Riyadh, Saudi Arabia \\ myshalaby@imamu.edu.sa
}

\author{
Mohamed Asad Ali \\ Electrical Engineering Department, \\ College of Engineering, Al Imam Mohammad Ibn Saud Islamic \\ University (IMSIU), Riyadh, Saudi Arabia \\ asadali@imamu.edu.sa
}

\author{
Mohammad Obaidullah Khan \\ Electrical Engineering Department, \\ College of Engineering, Al Imam Mohammad Ibn Saud Islamic \\ University (IMSIU), Riyadh, Saudi Arabia \\ OKKhan@imamu.edu.sa
}

\begin{abstract}
This paper introduces detection techniques of target gas concentration based on infrared absorption spectrum method and the simulation of the gas detection system using LabVIEW. In this work, the harmonic detection is performed by wavelength modulation technique with a related frequency signal to cover the measured signal and get the gas absorption coefficient that can be analysed to give a gas concentration. A series of simulations are carried out to scan weak absorption lines and extract the second harmonic curve of the absorption spectrum of the target gas. The absorption and second harmonic curves are acquired by changing the parameters of the target gas concentration and line width. This work claims to be a contribution to the study and the comprehension of gas detection system based on tunable diode laser absorption spectroscopy and can serve as a basis for practical data acquisition.
\end{abstract}

Keywords-infrared absorption spectrum; simulation system; second harmonic; phase-locked amplifier; TDLAS; LabVIEW

\section{INTRODUCTION}

Tunable diode laser absorption spectroscopy (TDLAS) [1, $2]$ is used to trace atmospheric constituent gases even with small concentrations as low as sub parts per billion (ppbv). The tunable diode laser source emits laser in mid infrared spectral region and has wavelengths from $3 \mu \mathrm{m}$ to $30 \mu \mathrm{m}$. By adjusting laser conditions, temperature or injection current, the output wavelength can be tuned even continuously in small intervals. Many trace gases strongly absorb the infrared spectrum. Tunable diode laser (TDL) has a line width of $10^{-4}$ to $10^{-5} \mathrm{~cm}^{-1}$ range. These conditions result in high resolution and have high selectivity. Second harmonic detection [3] is employed by using a modulation waveform in $\mathrm{kHz}$ frequency domain, this is simultaneously superimposed on the diode laser scanning current. A lock amplifier is used to detect the resultant frequency-modulated absorption. In this research the spectrum modulation method is chosen since the direct absorption method [4, 5] provides a very weak signal. The use of wavelength modulation spectroscopy (WMS) can clearly diminish the noise produced by the laser control and highly improve SNR. The injection current in the system is varied simultaneously with the output wavelength characteristic of TDL. The superimposition of the high-frequency sinusoidal current and the low-frequency sawtooth can drive the output wavelength scanning around the center wavelength of the laser. Realizing the wavelength modulation spectroscopy, the second harmonic and the absorption curves can be detected by the phase locked amplifier. Overall, this work which includes both theoretical aspects and simulations, claims to be a contribution to the study and understanding of gas detection in the infrared absorption spectrum

\section{TARGET GAS DETECTION}

Based on Lambert-Beer law, the harmonic detection method has a high sensitivity, high resolution, and fastresponse trace gas detection $[1,5]$. Near-infrared distributed feedback (DFB) semiconductor laser light source has a narrow bandwidth and rapid tuning characteristics. The detection of the gas concentration is done by detecting an isolated absorption line of the target gas. The applied light source spectrum contains the wavelength range of one or more gas absorption lines, when the light passes through the gas, attenuation occurs. The Lambert-Beer law is expressed by $(1)$ :

$$
I=I_{0} \exp (-\alpha C L)
$$

where $I$ is the transmission line intensity absorbed by the gas, $I_{0}$ is the incident light intensity, $\alpha$ is the gas absorption coefficient, $C$ is the gas concentration, and $L$ is the length of light through gas. 
At atmospheric pressure the molecular absorption line broadening is mainly pressure broadening, the linear absorption coefficient $\alpha$ can be approximated by the Lorentz law:

$$
\alpha(v)=N_{\mathrm{o}} \sigma(v)=\frac{N_{\mathrm{o}} \sigma\left(v_{\mathrm{o}}\right)}{1+\left(\frac{v-v_{\mathrm{o}}}{\gamma}\right)^{2}}
$$

where $N_{\mathrm{o}}$ is the number of molecules per unit volume at $25^{\circ}$ under atmospheric pressure. $N_{0}=2.6875 \times 10^{19}, \sigma(v)=$ cross section, $v=$ wave number, $v_{\mathrm{o}}=$ wave number at maximum absorption intensity, and $\gamma=$ half width at half maximum (HWHM). The detection system involves harmonic detection modeled and elaborated in the next section.

\section{IMPLEMENTATION AND MODELING}

\section{A. LabVIEW Based Detection System}

The target gas detection system has been implemented and simulated using LabVIEW (laboratory virtual instrument engineering workbench) which is a system-design platform and development environment. It is a graphical programming language which uses graphical user interface. It is simple and user friendly, a user can select a block from its function library and define the parameters, inputs and outputs and model a system. Subprograms can be defined as sub-VI, these sub-VI's can be connected at any stage, added, removed, varied and adjusted or modified as per the system modeled tested and remodeled according to task parameters changes with time and requirement. LabVIEW integrates the creation of user interfaces (termed front panels) into the development cycle. LabVIEW programs-subroutines are termed as virtual instruments (VIs). Each VI has three components: a block diagram, a front panel, and a connector panel. The last is used to represent the VI in the block diagrams of other, calling VIs. The front panel is built using controls and indicators. Controls are inputs: they allow a user to supply information to the VI. Indicators are outputs: they indicate, or display, the results based on the inputs given to the VI. The back panel, which is a block diagram, contains the graphical source code. All the objects placed on the front panel will appear on the back panel as terminals. The back panel also contains structures and functions which perform operations on controls and supply data to indicators. Collectively controls, indicators, structures, and functions are referred to as nodes. Nodes are connected to one another using wires, e.g. two controls and an indicator can be wired to the addition function so that the indicator displays the sum of the two controls. Thus a virtual instrument can be run as either a program, with the front panel serving as a user interface, or, when dropped as a node onto the block diagram, the front panel defines the inputs and outputs for the node through the connector panel. This implies each VI can be easily tested before being embedded as a subroutine into a larger program. The LabVIEW apart from providing programming can easily be integrated to direct hardware without any interface programming or special requirement by using the DAC (data acquiring and controlling) assistant to different interfacing boards $[14,15]$.

A thorough analysis of gas chamber parameters can be done by LabVIEW while a change with other parameters for say concentration and target gases can get the results for second harmonic and absorption curves. An analysis for different ramp laser light range can be done to get different target gases. Different modulation coefficients can be adjusted to get weak signals and a thorough analysis can be done. A typical frame of the model for detection of target gas using LabVIEW is illustrated in Figure 1.

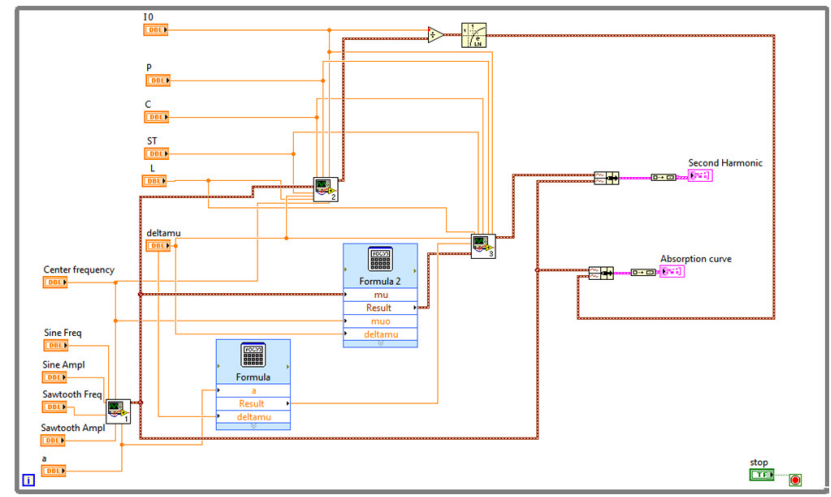

Fig. 1. A typical frame of a virtual system for target gas detection on LabVIEW platform

This model works on harmonic detection technique. Wavelength modulation and second harmonic signal detection (2f) are both used in harmonic detection [6] to achieve highly sensitive target gas detection. The wavelength modulation of absorption spectra can produce a harmonic signal which is directly proportional to the concentration of trace gases. This is called zero background spectrum detection technique [3, 4]. To produce the harmonic signal by wavelength modulation a cosine modulation of angular frequency $\omega$ is superimposed on laser (sawtoth) which results in time varying laser frequency that can be given by:

$$
V=V_{c}+A \operatorname{Cos}(\omega t)
$$

where $V$ is the instantaneous frequency of the laser wavelength, $V_{c}$ is the center frequency of laser modulation, and $A$ is the modulation depth.

The wavelength of the laser output is a modulated cosine, then the signal generated by passing laser through the gas is a periodic even function which can be expanded in a cosine Fourier series as expressed in (4):

$$
S=\sum_{n=0}^{\infty} H n(\mathrm{Vc}) \cos (n \omega t)
$$

where $H n$ is the $n$th harmonic component of the modulated absorption coefficient. If the absorption coefficient $\alpha$ is sufficiently small, $H n$ can be approximated to $H n \propto I_{o} \alpha L C$

The above harmonic components are proportional to the gas concentration and the amplitude of each harmonic decreases when the number of harmonics increases. Low harmonic is chosen to obtain higher SNR and by using a lock in amplifier a harmonic component can be selected.

\section{B. Typical Principle Model of Harmonic Detection}

A typical schematic block diagram of wavelength modulation is illustrated in Figure 2. 


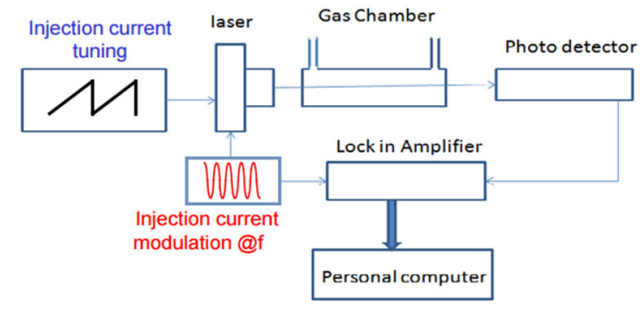

Fig. 2. Schematic model of wavelength modulation spectroscopy

In the gas concentration system, the received laser signal is not unlimited and the component of the harmonic signal is weak. The band pass filter method can filter out the white noise. This means that the width of the filter is very thin, which is not good because the quality factor $Q$ is generally less than 100. Consequently, the curve of the amplitude-frequency characteristic is not smooth enough and varies nonlinearly in the pass-band [7]. Lock-in amplifier method is used instead to remove the external noise and extract a high-precision harmonic signal. The basic principles of the lock-in amplifier are performed by wavelength modulation like sinusoidal modulation with a related frequency signal and the signal is scanned to cover the measured signal. The modulation frequency is taken as a reference signal to the input into the locked-in amplifier. The locked-in amplifier technique extracts the harmonics signals at the same frequency, gives good performance in terms of density signal extraction, and improves significantly the detection SNR for weak signals [8]. As illustrated in Figure 2, an analog locked-in amplifier is used in the detection of trace signals because the error of harmonic amplitude signal extracted is smaller [9]. With the new DSP technology [10-12], a digital lock-in amplifier is used which can convert analog to digital signals. The center frequency of light output is accurately locked on the gas absorption peak. That means $V_{0}=V_{\mathrm{c}}$. The detected signal can be obtained as:

$$
I=I_{0}\left[1+n \sin \omega t-\frac{\alpha 0 c L}{1+\omega^{2} \sin ^{2} \omega t}\right]
$$

where $\omega=\frac{v f}{\Delta v}, I_{0}$ is the input signal amplitude, $n$ is the light intensity coefficient, $c$ is the measured gas concentration, $L$ is the effective optical path, $v f$ is the frequency modulation amplitude and $\Delta v$ is HWHM.

Equation (5) can be expanded into a Fourier series sequence, since the higher harmonics' amplitude is too small to be ignored. The second harmonic coefficients are shown in (6):

$$
I_{2 f}=-\frac{2\left[2+\omega^{2}-2\left(1+\omega^{2}\right)^{0.5}\right]}{\omega^{2}\left(1+\omega^{2}\right)^{0.5}} \alpha_{\mathrm{o}} c L I_{\mathrm{o}}
$$

From (6) it is inferred that the amplitude of the second harmonic signal intensity is related to the initial gas concentration. Thus, by extracting the second harmonic signal, gas concentration information can be retrieved. The locked-in amplifier method has the advantages of minor measurement error, big dynamic range, and no temperature bias effects [11].

\section{SimUlation RESUlTS AND DISCUSSION}

The detection system is characterized by initial characteristics [16] such as the optical path with a length of $L$, the incident intensity $I_{0}$, the width of line $\Delta v$, and the target gas concentration $C$. The values of these parameters are: $I_{0}=100 \mathrm{~cd}$ and $C$ value is changed from $10 \%$ to $100 \%$. The line width $\Delta v$ is changed from 0.01 to 0.09 and $L=15 \mathrm{~m}$. Figure 3 illustrates the simulation of the spectrum of gas absorption in terms of the scanning frequency. Figure 4 shows the second harmonic wave of the emission output intensity in terms of scanning frequency.

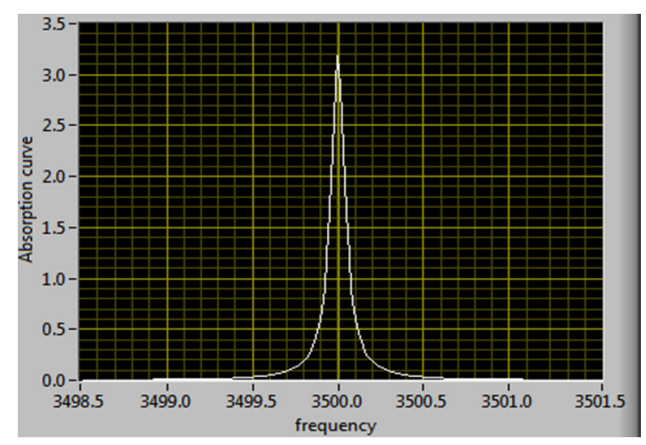

Fig. 3. Absorption curve vs concentration of the target gas specimen

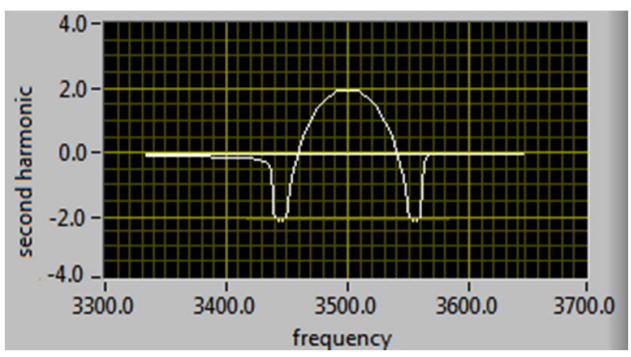

Fig. 4. Second harmonic wave vs concentration of the target gas specimen

It can be observed that the target gas absorption amplitude and the second harmonic depend on gas concentration. Figure 5 shows that the gas concentration is proportional to the absorption peak values. As the concentration of gas gets smaller, the output intensity of the laser beam gets higher and the degree of absorption of the optical chamber decreases. In Figure 6 the concentration of the gas is exhibited as proportional to the peak values of the second harmonics. As the gas concentration gets higher, the value of the second harmonic peak gets higher also. As the concentration gets higher, the probability of detecting the gas increases. Similarly, the values of the other initial detection system parameters can similarly affect the gas absorption curve and the second harmonic wave. Figure 6 depicts that by varying the value of the modulation coefficient $\Delta v$ over an interval of 0.01 to 0.09 , and as the line width increases the broadening coefficient collision $\Upsilon$ gets larger and the peak curve becomes stout and the absorption peak height gets smaller. Figure 7 shows that as the line width increases the broadening coefficient collision $\Upsilon$ gets larger, this includes the peak curve becoming stout and then the $\mathrm{I} 2 \mathrm{f}$ peak height gets smaller. As the line width increases the band width increases. Due to this effect the curve becomes of less height (amplitude) in value and more wide (bandwidth increases with frequency range). This is an advantage when the target gas has narrow spectra. The line width can be increased, the frequency range will be broader and then the target gas spectra can be easily detected. 


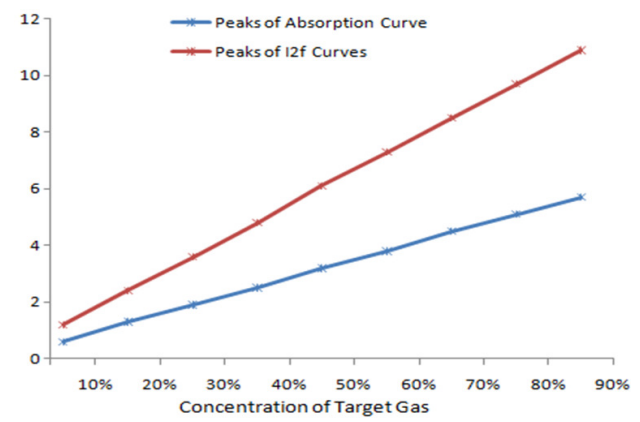

Fig. 5. Peaks of absorption and second harmonic curves vs concentration of the target gas specimen

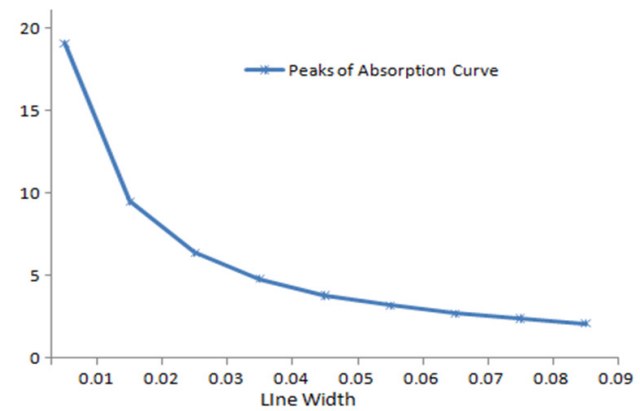

Fig. 6. Peaks of absorption curves vs line width

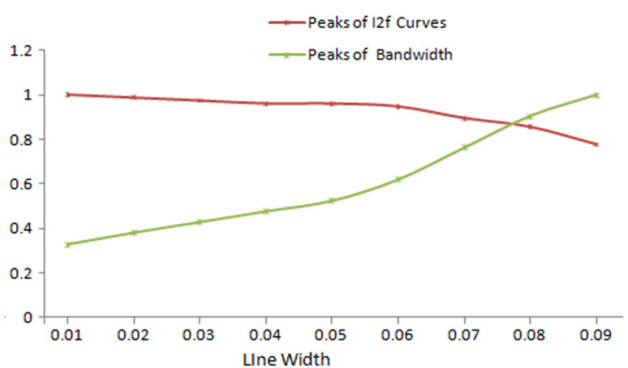

Fig. 7. Peaks of bandwidth and second harmonic curves vs line width

\section{CONCLUSION AND FUTURE SCOPE}

This paper provides a detection method of the target gas modulated weak signal, by which concentration levels can be determined for different gases. LabVIEW simulation and analysis illustrated that the superimposition of the high frequency sinusoidal current and the low frequency saw-tooth current can provide the tuning of output wavelength around the central frequency and then the second harmonic and the absorption curves are detected by the phase locked amplifier that is proportional to the gas concentration within a certain range. The developed model and analysis can be thought of as a basis for practical gas detection systems and can also be applied for magnetic resonance imaging systems or other areas of spectroscopy.

\section{ACKNOWLEDGMENT}

The authors would like to thank the deanship of Academic Research at Al Imam Mohammad Ibn Saud Islamic University for the financial support of this project.

\section{REFERENCES}

[1] L. Shuqin, The Analysis and Detection System of $\mathrm{CO} 2$ Gas Based on TDLAS, Harbin Industrial University, 2013 (in Chinese)

[2] L. Ning, W. Fei, Y. Jianhua, M. Zengyi, C. Kefa, "Gas concentration measuremen based on tunable semiconductor laser absorption spectrum technology", Proceedings of the CSEE, Vol. 25, No. 15, pp. 121-126, 2005

[3] J. L. Han, L. Jianguo, Y. B. He, J. F. He, L. Yau, Z. Yu, J. Ying, S. Yuan, R. F. Kan, "Simulation and analysis of second harmonic signal of the tunable diode laser absorption spectroscopy", Spectroscopy and Spectral Analysis, Vol. 33, No. 4, pp. 881-885, 2013 (in Chinese)

[4] G. Lijun, Research on Methane Detection Based on the Principle of Near Infrared Absorption, Hebei Yanshan University, 2008 (in Chinese)

[5] S. Hui, Multi-component gas analyzer based on infrared absorption theory, Harbin Industrial University, 2009 (in Chinese)

[6] L. Tongxing, L. Yiqun, The principle and application of laser spectroscopy, Anhui University of Science and Technology of China Press, 2006 (in Chinese)

[7] X. F. Nan, Y. Zhang, M. Yu, "Laser gas telemetry test system automation test software design", Optical Instruments, Vol. 37, No. 3, pp. 210-213, 2016

[8] M. L. Cong, D. D. Sun, Y. D. Wang, “Application of Logarithmic Transformation-Wavelength Modulation Spectroscopy in Gas Detection", Infrared and Laser Engineering, Vol. 46, No. 2, pp. 1-6, 2017

[9] A. De Marcellis, G. Ferri, A. D’Amico, C. Di Natale, E. Martinelli, “A fully-analog lock-in amplifier with automatic phase alignment for accurate measurements of ppb gas concentrations", IEEE Sensors Journal, Vol. 12, No. 5, pp. 1377-1383, 2012

[10] S. M. Ahmed Ghaly, S. S. Al-Sowayan, "A high B1 field homogeneity generation using free element elliptical four-coil system", American Journal of Applied Sciences, Vol. 11, No. 4, pp. 534-540, 2014

[11] S. M. Ahmed Ghaly, K. A. Al-Snaie, S. S. Al-Sowayan, "Design and Testing of Radiofrequency Spherical Four Coils", Modern Applied Science Jounal, Vol. 10, No. 5, pp. 186-193, 2016

[12] S. M. Ahmed Ghaly, K. A. Al-Snaie, O. K. Mohammad, "Spherical and Improved Helmholtz Coil with High B1 Homogeneity for Magnetic Resonance Imaging”, American Journal of Applied Sciences, Vol. 13, No. 12, pp. 1413-1418. 2016

[13] H. F. Liu, B. Li, Q. X. He, J. M. Dang, H. Y. Yu, Z. T. Zheng, Y. D. Wang, J. Q. Pan, "Development of digital orthogonal lock-in amplifier and its application in methane detection", Acta Photonica Sinica, Vol. 45, No. 4, pp. 0423004-1 - 0423004-6, 2016 (in Chinese)

[14] C. J. Kalkman, "LabVIEW A software system for data acquisition, data analysis, and instrument control", Journal of Clinical Monitoring, Vol. 11, No. 1, pp. 51-58, 1995

[15] A. Gani, M. J. E. Salami, "A LabVIEW based data acquisition system for vibration monitoring and analysis", Student Conference on Research and Development, Shah Alam, Malaysia, July 17, 2002

[16] Y. Huo, W. Fan, "Application of harmonic detection technology in methane telemetry", Journal of Physics: Conference Series, Vol. 887, ArticleID 012020, 2017 